\title{
UV/OPTICAL CONTINUUM VARIABILITY IN AGNS
}

\author{
WEI-HSIN SUN \\ Institute of Astronomy, National Central University, \\ Taiwan, ROC \\ CHARLENE A. HEISLER \\ Anglo-Australian Observatory, Epping Laboratory, Australia \\ AND \\ MATTHEW A. MALKAN \\ Department of Astronomy, UCLA, USA
}

\section{Introduction}

The observed strong UV/Optical excess in many Seyfert 1 galactic nuclei and quasars (QSOs) is well described as thermal radiation from the surface of an optically thick accretion disk surrounding a supermassive black hole (Malkan 1983, Sun and Malkan 1989). This scenario naturally leads to a radially symmetric temperature gradient with the innermost regions the hottest. Thus for a perturbation generated in the inner regions propagating outward, sequential variations from higher to lower frequencies should be expected, aside from the bolometric increase of brightness. However, the extremely intensive International AGN watch (Peterson 1993 and references therein) on NGC 5548 and UV/Optical monitoring campaign on Mkn 335 (Sun et al. 1995) point to opposite conclusions on the time lag between UV and Optical continua, with no lag for NGC 5548 and $\sim 50$ days for Mkn 335. We thus began two monitoring programs at Lick Observatory and Mount Stromlo and Siding Spring Observatory (MSSSO) on a sample of Seyfert 1 galaxies with CCD direct imaging to search for more conclusive evidence on the sequential variabilities.

\section{Observations}

The variabilities of the target AGNs at $3890 \AA$ (U), $5750 \AA$ (V), $8020 \AA(\mathrm{I})$, and $9750 \AA(\mathrm{Z})$, were measured with two-inch square intermediate band 


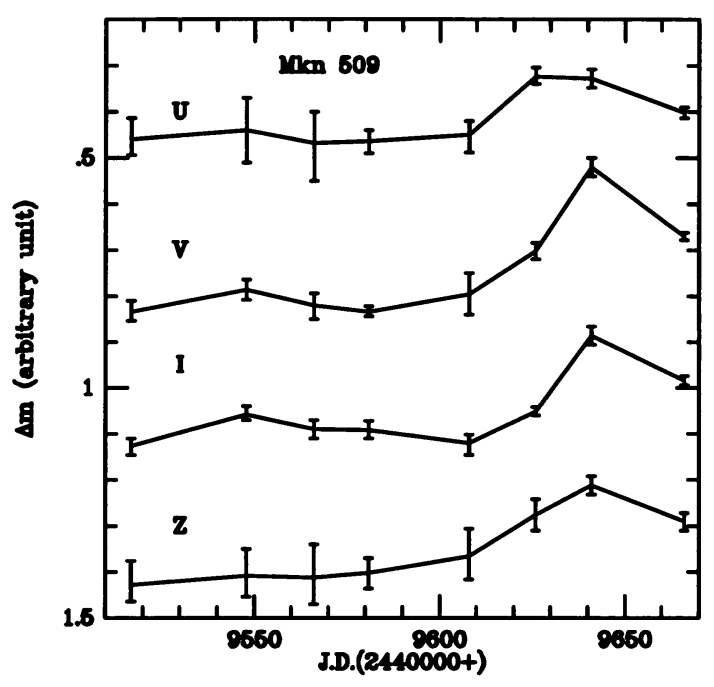

Figure 1. Four-color light curves of Mkn 509

$(250-300 \AA)$ filters via differential photometry with non-variable field stars at the 1-m telescopes at Lick and MSSSO. The observations are carried out approximately once every month, starting June 1994 at Lick and October 1994 at MSSSO.

\section{Preliminary Results}

We find non-simultaneous variabilities in the four selected wavebands on Mkn 509 with Lick Observations combined with MSSSO data (from 6/94 through 11/94). Three interesting points can be inferred: (i) the variability amplitudes are in general $0.15-0.20$ magnitudes in all four filters over the 6 month period, while the typical error bars are 0.04 magnitude, thus allowing the variability to be accurately measured; (ii) the observed variations in each filter are not in phase, suggesting the possibility of sequential variation in neighboring wavebands; and (iii) considerable color change from $\mathrm{U}$ through $\mathrm{Z}$ in 6 months is observed. While the current dataset show interesting implications in understanding how the optical continuum in AGN varies, we still need more data points for rigorous time analysis.

\section{References}

Malkan, M.A., 1983, ApJ, 275, 477

Peterson, B.M., 1993, PASP, 105 (685), 247

Sun, W.-H. \& Malkan, M.A., 1989, ApJ, 346, 68

Sun, W.-H. et al., 1995, in preparation 